\title{
TRATAMIENTO DE LA DISFUNCIÓN ERÉCTIL MEDIANTE EL IMPLANTE DE PRÓTESIS DE PENE. ANÁLISIS DE NUESTRA SERIE
}

\author{
Ó. RODRÍGUEZ FABA, J.L. MARTÍN BENITO, S. ESCAF BARMADAH, \\ J.J. RODRÍGUEZ MARTÍNEZ, A. JALÓN MONZÓN, J. REGADERA SEJAS
}

Servicio de Urología I. Hospital Universitario Central de Asturias. Oviedo. Asturias.

Actas Urol Esp. 28 (8): 575-580, 2004

\section{RESUMEN}

TRATAMIENTO DE LA DISFUNCIÓN ERÉCTIL MEDIANTE EL IMPLANTE DE PRÓTESIS DE PENE. ANÁLISIS DE NUESTRA SERIE

INTRODUCCIÓN Y OBJETIVOS: El objetivo de este estudio fue revisar la situación actual de las prótesis de pene en el tratamiento de la disfunción eréctil; así como analizar las indicaciones, el grado de aceptabilidad y las complicaciones en nuestra experiencia.

MATERIAL Y MÉTODOS: Entre 1984 y 2003 implantamos 48 prótesis de pene en varones con edad media de 55 años. Los antecedentes patológicos más frecuentes fueron: enfermedad vascular, diabetes mellitus y cirugía pélvica. El tipo de prótesis utilizada fue inactiva maleable en 7 casos $(14,58 \%)$, Jonas ${ }^{6}$, Acu-form ${ }^{1}$. Activa inflable o hidráulica de 3 componentes en 19 casos $(39,58 \%)$, AMS 700 plus ${ }^{17}$, Alpha ${ }^{2}$. Activa inflable, integrada o autocontenida en 5 casos (10,41\%), Hydroflex ${ }^{4}$, Dynaflex ${ }^{1}$. Activa inflable de 2 piezas en 17 casos $(35,4 \%)$, Ambicor ${ }^{2}$, Mark II ${ }^{15}$.

RESULTADOS: El 6,25\% presentó infección protésica que obligó a retirar el implante, y el 4,16\% complicaciones mecánicas. El $80 \%$ de los pacientes se mostraron satisfechos con la prótesis.

CONCLUSIONES: Las prótesis peneanas continúan siendo una alternativa válida y eficaz en el tratamiento del varón con disfunción eréctil, presentando un bajo índice de complicaciones.

PALABRAS CLAVE: Disfunción eréctil. Prótesis de pene. Impotencia.

\section{ABSTRACT}

TREATMENT OF ERECTILE DYSFUNCTION WITH PENILE PROTHESIS. OUR EXPERIENCE

INTRODUCTION AND OBJETIVES: The aim of this study was to review the current situation of penile prosthesis in the treatment of erectile dysfunction and to analyze the indications, degree of acceptability and complications in oir experience.

MATERIAL AND METHODS: From 1984 to 2003, 48 implants were inserted in men suffering from impotence due to different etiologies. The mean age was 55 years. Vascular disease, Diabetes and pelvic surgery were the most common pathologies recorded. The type of prothesis inserted were malleable inactive in 7 cases (14.58\%), Jonas ${ }^{6}$, Acu-form ${ }^{1}$. Inflable active with 3 components in 19 cases (39.58\%), AMS 700 plus $^{17}$, Alpha ${ }^{2}$. Inflable active integrated in 5 cases (10.41\%) Hydroflex ${ }^{4}$, Dynaflex ${ }^{1}$. Inflable active with 2 components in 17 cases (35.4\%), Ambicor ${ }^{2}$, Mark $\mathrm{II}^{15}$.

RESULTS: Infection of the prosthesis was observed in $6.25 \%$ of the cases. This infectious complication required removal of the prosthesis. $4.16 \%$ of the cases complained of mechanical failure that required change of the prosthesis. $80 \%$ of the patients were satisfied with the results.

CONCLUSIONS: Penile prostheses continue to be an effective and valid therapeutic alternative in impotent. Although the complication rate is low, adequate information must be provided to the patient. KEY WORDS: Erectile dysfunction. Penile prosthesis. Impotence. 
$\mathrm{E}^{1}$ objetivo de este estudio ha sido analizar la situación actual del implante de prótesis de pene para el tratamiento de la disfunción eréctil a través de la revisión retrospectiva de nuestra serie.

Históricamente, la primera aproximación a la cirugía protésica del pene se realizó mediante la colocación de un cartílago costal dentro de un injerto tubulizado para la reconstrucción peneana tras amputación traumática.

En 1952 Goodwin y Scott utilizaron prótesis acrílicas extracavernosas con mal resultado, ya que sufrían fácilmente extrusión o perforación cutánea. Posteriormente en 1960, Pearlman y Lash Loeffler colocaron dispositivos rígidos de una sola pieza que se implantaban debajo de la fascia en el eje del pene, más tarde Beheri inició la colocación de prótesis intracavernosas de polietileno.

La era actual comienza con la colocación de las primeras prótesis por Small y Carrión en 1973, mejoradas por Finney en 1977 mediante la introducción de su charnela para una mejor adaptación, más tarde Jonas la mejora con su ánima de plata que facilita la maleabilidad.

Las prótesis inflables intracavernosas también fueron introducidas en 1973 por Scott ${ }^{1,2}$.

Actualmente dispositivos que más se son los semirrígidos y los inflables que elaboran fundamentalmente AMS y Mentor. Las prótesis de varilla de Mentor son la Acu Form y la Mentor Malleable. AMS tiene una prótesis de varilla, la AMS Malleable 650. Las prótesis inflables de dos piezas de Mentor son la Mark II y la equivalente en AMS es la Ambicor. Las prótesis de tres piezas incluyen la Alpha I y la Alpha I Narrow Base de Mentor, y la $700 \mathrm{CXM}$, la $700 \mathrm{CX}$ y la Ultrex, las tres de AMS ${ }^{1}$.

En 1998 el citrato de Sildenafilo se convierte en la primera línea de tratamiento para la mayoría de los hombres con $\mathrm{DE}$ y cuando estas terapias orales actúan, por lo general no se piensa en otras líneas para el tratamiento de la DE.

Cuando fracasan la mayoría de los hombres necesitan información sobre los dispositivos de erección por vacio, fármacos intrauretrales, inyecciones peneanas e implante de prótesis.

La elección del implante de prótesis puede ser por el fracaso en el resto de alternativas a la terapia oral, o porque estas alternativas son inaceptables para el paciente y su pareja.

Revisamos, a través de nuestra serie, la evolución histórica de las indicaciones de implante antes y después de la aparición de fármacos orales. También repasamos la evolución técnica y quirúrgica de los implantes.

\section{MATERIAL Y MÉTODOS}

Entre 1984 y 2003 se implantaron en nuestro servicio un total de 48 prótesis de pene en pacientes con edades comprendidas entre los 25 y 71 años de edad. La edad media de los pacientes era de 55 años.

Del total de pacientes $33(68,75 \%)$ recibieron el implante antes de la aparición en el mercado de citrato de sildenafilo siendo la etiología vascular en 23 casos $(69,6 \%)$, causas quirúrgicas (prostatectomía $\mathrm{u}$ otras) en 2 casos $(6,06 \%)$, enfermedades del pene en 2 casos (6,06\%), enfermedades neurológicas en 5 casos $(15,15 \%)$, traumatismos pélvicos en 1 caso $(3,03 \%)$.

Los restantes 15 pacientes $(31,25 \%)$ recibieron el implante después de la aparición del citrato de sildenafilo, siendo la etiología vascular en 9casos (60\%), causas quirúrgicas (prostatectomía radical) en 5 casos $(33,3 \%)$ y enfermedades del pene en 1 caso $(6,6 \%)$ (Tablas 1 y 2 ).

\section{Tabla 1}

Indicación antes de la aparición de Sildenafilo

\begin{tabular}{lrc}
\hline Total & 33 & $68,75 \%$ \\
Vascular & 23 & $69,6 \%$ \\
Cirugia & 2 & $6,06 \%$ \\
Enf. del pene & 2 & $6,06 \%$ \\
Enf. neurol. & 5 & $15,15 \%$ \\
Traumat. & 1 & $3,03 \%$ \\
\hline
\end{tabular}

Tabla 2

Indicación después de la aparición del Sildenafilo

\begin{tabular}{lrl}
\hline Total & 15 & $31,25 \%$ \\
Vascular & 9 & $60 \%$ \\
Cirugia & 5 & $33,3 \%$ \\
Enf. pene & 1 & $6,6 \%$
\end{tabular}


Todos los pacientes fueron estudiados con el fin de determinar la etiología de su disfunción eréctil orgánica mediante un estudio hormonal que incluye testosterona libre matutina (la determinación de FSH y LH se realizó solamente cuando la testosterona presentaba niveles disminuidos) y prolactina, Eco-doppler arterial y test de drogas vasoactivas realizado con 20 microgramos de PGE1.

En el momento del implante descartamos la existencia de infección de las vías urinarias, infecciones sistémicas y lesiones potencialmente infectadas en la zona operatoria.

Una hora antes del procedimiento administramos de forma profiláctica $1 \mathrm{G}$ de Ampicilina y 80 MG de Gentamicina, y si el paciente es alérgico a la Penicilina y derivados sustituimos la Ampicilina por $1 \mathrm{G}$ de Vancomicina. El rasurado de la zona operatoria se realiza justo antes de la cirugía. Mantenemos la antibioterapia por vía venosa hasta el día siguiente del procedimiento.

$\mathrm{El}$ abordaje se puede hacer por una incisión perineal, peneano-escrotal, subpúbica, transcorporal y subcoronal, nosotros lo hacemos mediante una incisión penoescrotal que nos proporciona una buena exposición de los cuerpos cavernosos en su porción media, realizamos una disección cuidadosa evitando hematomas, abordamos la albugínea a través de la fascia de Buck abriéndola $3 \mathrm{~cm}$ longitudinalmente; nosotros colocamos dos puntos de referencia a cada lado de la incisión antes de dilatar los cuerpos cavernosos, una vez dilatados, medida la longitud y colocada la prótesis, cerramos la cavernotomía con puntos sueltos de una sutura reabsorbible (Vicril $2 / 0$ ). Habitualmente dejamos un drenaje aspirativo en escroto y una sonda vesical durante 24 horas. En las prótesis que requieren colocar reservorio, este lo colocamos mediante una pequeña incisión suprapúbica de $5 \mathrm{~cm}$ dejando el reservorio en el espacio de Retzius.

El tipo de prótesis utilizada fue inactiva maleable en 7 casos $(14,58 \%)$, Jonas ${ }^{6}$, Acu-form ${ }^{1}$. Activa inflable o hidráulica de 3 componentes en 19 casos (39,58\%), AMS 700 plus $^{17}$, Alpha ${ }^{2}$. Activa inflable, integrada o autocontenida en 5 casos $(10,41 \%)$, Hydroflex ${ }^{4}$, Dynaflex ${ }^{1}$. Activa inflable de 2 piezas en 17 caso $(35,4 \%)$, Ambicor $^{2}$, Mark II ${ }^{15}$ (Tabla 3).
Tabla 3

Tipos de prótesis implantadas

\begin{tabular}{lcll}
\hline $\begin{array}{l}\text { Inactiva } \\
\text { maleable }\end{array}$ & $7(14,58 \%)$ & Jonas $^{6}$ & Acuform $^{1}$ \\
$\begin{array}{l}\text { Activa } \\
\text { inflable de } 3 \\
\text { componentes }\end{array}$ & $19(39,58 \%)$ & ${\text { AMS 700 } \text { plus }^{17}}$ & Alpha $^{2}$ \\
$\begin{array}{l}\text { Activa } \\
\text { inflable de 2 } \\
\text { piezas }\end{array}$ & $17(35,4 \%)$ & Ambicor $^{2}$ & Mark II $^{15}$ \\
$\begin{array}{l}\text { Activa } \\
\text { inflable } \\
\text { autocontenida }\end{array}$ & $5(10,41 \%)$ & Hydroflex $^{4}$ & Dynaflex $^{1}$ \\
\hline
\end{tabular}

$\mathrm{Al}$ alta se le enseña al paciente a manipular la prótesis, que recomendamos comenzar a usar 6 semanas después de la cirugía, programándose visitas inicialmente semestrales y posteriormente anuales.

\section{RESULTADOS}

El objetivo ideal de la colocación de la prótesis nos da como resultado un pene que se parezca al normal en cuanto a flacidez y a erección para permitir una adecuada penetración sin cambios en la eyaculación ni en el orgasmo.

En nuestra serie hemos detectado las siguientes complicaciones que dividimos en per-operatorias, post-operatorias inmediatas y post-operatorias tardías ${ }^{3}$.

\section{Complicaciones per-operatorias}

Evitamos en lo posible durante el transcurso de la intervención que se produzcan hematomas ya que dificultan la cicatrización y favorecen las infecciones.

Hemos detectado en un caso perforación del tabique pectiniforme que nos ha obligado a realizar la colocación de los cilindros con sumo cuidado para evitar la inserción de los dos en un solo cuerpo cavernoso, a pesar de ello utilizamos la tijera para la dilatación inicial de los cuerpos cavernosos en lugar del cavernotomo de Carrión-Roselló.

No hemos tenido ningún caso de perforación del cono proximal que nos obligaría a realizar un fruncimiento por vía perineal.

En 3 casos hemos tenido perforación del cono distal del cuerpo cavernoso esperando 2 meses para colocar la prótesis definitivamente ya que la sutura inicial de esta zona nos condujo en una ocasión a la extrusión de la prótesis. 
En un caso hemos tenido perforación vesical durante la disección de los espacios laterovesicales para colocar el reservorio. Este paciente presentaba el antecedente de cirugía radical de próstata y precisó sutura vesical y mantener la sonda durante 15 días (Tabla 4).

\section{Tabla 4}

Complicaciones per-operatorias

\begin{tabular}{ll}
\hline Perforación del tabique pectiniforme & $1(2,08 \%)$ \\
Perforación del cono proximal & 0 \\
Perforación del cono distal & $3(6,25 \%)$ \\
Perforación vesical & $1(2,08 \%)$ \\
\hline
\end{tabular}

Complicaciones post-operatorias inmediatas (24-48 horas)

No hemos tenido casos de retención urinaria ya que por norma colocamos una sonda Foley CH16 al iniciar la cirugía y la mantenemos durante 24 horas.

En 7 casos hemos detectado hematomas cuantiosos que no han precisado drenaje y se han resuelto con protección antibiótica de forma conservadora. En uno de estos casos el hematoma se localizó en el espacio de Retzius que ocasionó un ileo reflejo precisando la colocación de sonda nasogástrica e ingreso hospitalario durante 7 días.

El dolor post-operatorio es común y se resuelve con analgesia de primer escalón pautada cada 8 horas hasta el alta.

No hemos tenido ningún caso de infección en las primeras 48 horas que nos obligase a retirar la prótesis de forma precoz.

\section{Complicaciones post-operatorias tardías}

En 6 casos el edema postoperatorio de la zona se ha mantenido durante 15 días- 1 mes, lo tratamos con anti-inflamatorios y se resuelve sin problemas cuando no es debido a infección.

Hemos tenido 2 casos de isquemia en glande tardía.

En 1 caso tuvimos dolor importante en el punto de flexión de la prótesis que se resolvió con analgésicos sin más problemas.

Hemos tenido en 1 caso rotura del vástago metálico que obligó a cambiar la prótesis.
En 2 casos rotura del sistema de bombeo con pérdida de líquido que también obligó a cambiar la prótesis.

Un caso de migración del reservorio intraescrotal hacia la región perineal que precisó recolocación.

Un caso de fístula escrotal en un paciente diabético severo e infección que obligó a la retirada de la prótesis.

Hemos tenido dos casos de infección de material protésico por Sthaphilococo epidermidis en pacientes lesionados medulares que nos han obligado a retirar la prótesis (Tabla 5).

Tabla 5. Complicaciones pos-operatorias tardias

\begin{tabular}{|c|c|c|}
\hline Edema & $\begin{array}{c}6 \\
(12,5 \%)\end{array}$ & AINES \\
\hline $\begin{array}{l}\text { Isquemia } \\
\text { Glande }\end{array}$ & $\begin{array}{c}2 \\
(4,16 \%)\end{array}$ & $\begin{array}{l}\text { Tratam. } \\
\text { conserv. }\end{array}$ \\
\hline Dolor & $\begin{array}{c}1 \\
(2,08 \%)\end{array}$ & AINES \\
\hline $\begin{array}{l}\text { Rotura } \\
\text { del vástago } \\
\text { metálico }\end{array}$ & $\begin{array}{c}1 \\
(2,08 \%)\end{array}$ & Recambio \\
\hline $\begin{array}{l}\text { Migración } \\
\text { reservor. }\end{array}$ & $\begin{array}{c}1 \\
(2,08 \%)\end{array}$ & Recambio \\
\hline $\begin{array}{l}\text { Fistula } \\
\text { escrotal }\end{array}$ & $\begin{array}{c}1 \\
(2,08 \%)\end{array}$ & Retirada \\
\hline Infección & $\begin{array}{c}3 \\
(6,25 \%)\end{array}$ & Retirada \\
\hline
\end{tabular}

\section{DISCUSIÓN}

La disponibilidad de una farmacoterapia eficaz para el tratamiento de la disfunción eréctil ha dado como resultado un notable incremento en la cantidad de hombres que se presentan para el tratamiento de su disfunción eréctil.

A los que fracasan en la terapia sistémica se les ofrecen terapias de segunda línea como dispositivos de vacío, medicación intrauretral e inyecciones intracavernosas.

Los hombres que están motivados se les propone la colocación de una prótesis después de fracasar los tratamientos de segunda línea ${ }^{1}$.

Colocamos prótesis en pacientes con D.E. de causa psicógena después de un tratamiento psicológico-psiquiátrico de al menos 2 años bien dirigido y sin resultados positivos, aunque de momento no hemos colocado ninguna prótesis en pacientes con este tipo de disfunción ${ }^{3}$. 
En caso de pacientes con enfermedad de Peyronie a pesar de que existe controversia sobre si es necesario la incisión sobre la placa, al igual que otros autores, somos partidarios de modelar el pene después del inflado de los cilindros sobre todo cuando implantamos prótesis CX de expansión circunferencial. Se permite una curvatura residual después del modelado de entre 20-30 grados.

Consideramos que la corporoplastia durante el implante de la prótesis alarga el tiempo operatorio, requiere la movilización de las bandeletas neurovasculares y si requiere la colocación de material sintético en el lugar de la placa aumenta el riesgo de herniación de un cilindro y de infección ${ }^{4}$.

En el caso de implantación de prótesis en cuerpos fibróticos, otros autores, sobre todo cuando ha existido una prótesis previa infectada, o después de priapismo o radioterapia, encuentran dificultades importantes para la dilatación y recomiendan iniciar la disección del cuerpo con tijera antes de introducir el dilatador Hegar de 8 $\mathrm{mm}$, siendo a veces necesaria la resección de parte del cuerpo fibroso ${ }^{1}$. En nuestra experiencia hemos colocado una prótesis en un paciente con secuela de priapismo sin objetivar durante la cirugía fibrosis de cuerpos que dificultase en exceso la dilatación, y en los recambios realizados en 3 ocasiones por fallos mecánicos solamente encontramos dificultades para extraer el sistema de bombeo en 1 caso en el que en la cirugía previa había existido un hematoma intraescrotal importante con fibrosis residual, sin tener problemas con la dilatación y el resto de la intervención en ningún caso ${ }^{2}$.

En cuanto a las indicaciones coinciden con las del resto de autores, ocupando la mayoría de ellas la enfermedad vascular severa, diabetes y cirugía. En nuestra serie resulta interesante destacar que antes de la aparición de el citrato de Sildenafilo colocábamos más prótesis (33 casos) con un predominio de pacientes con enfermedad vascular $(69,6 \%)$.

Después de la aparición del Sildenafilo colocamos globalmente menos prótesis(15 casos), la indicación vascular es ligeramente menor (60\%), y ha aumentado de forma importante 33,3\% de casos frente al 6,06\% previo la indicación por cirugía previa, sobre todo por prostatectomía radical.
Utilizamos la Ampicilina asociada a un aminoglucósido con profilaxis para minimizar el riesgo de infección, aunque otros utilizan de entrada vancomicina y aminoglucosido aunque el paciente no sea alérgico a penicilina y derivados ${ }^{5}$.

Utilizamos como vía de abordaje la peno escrotal realizando una incisión suprapúbica para colocar el reservorio.

La vía peno escrotal tiene la ventaja de presentar menor morbilidad y menor tiempo quirúrgico, y presenta la ventaja de la imposibilidad virtual de lesionar el nervio dorsal que es una complicación sin solución posible que deja el pene sin sensibilidad.

La complicación más grave que hemos tenido es la infección en 3 casos (6,02\%) en línea con el intervalo del $2-12 \%$ de la mayoría de autores (Scott 2\%, Kabalin 2,2\%, Anafarta 4,16\%, Thomalla 8,3\%, Mulcany $12 \%)^{6-8}$.

Especialmente importante es la tasa de infecciones en prótesis implantadas en lesionados medulares. Kimono y cols. presentaron una serie de 82 pacientes lesionados medulares entre 14 y 67 años en los que se colocó prótesis para el manejo de disfunciones urinarias por vejiga neurógena y disfunción eréctil en la que refieren una tasa de complicaciones global del $13,3 \%$ con un $10 \%$ de infecciones, sobre todo por gérmenes gram positivos, el principal argumento de la alta tasa de infección lo basan en el uso de antibióticos inapropiados para gérmenes gram positivos .En nuestra experiencia en los 2 casos de lesionados medulares que hemos colocado prótesis, hemos tenido infección-extrusión con retirada de la misma en ambos $\operatorname{casos}^{9}$.

También es importante resaltar el importante aumento de la tasa de infección en pacientes diabéticos. En nuestra serie de los 3 pacientes que presentaron infección, uno era diabético, y algunos autores como Lynch publican una tasa de infección del $22 \%$.

De forma general el germen más habitual que encontramos es el Staphilococo epidermidis que gracias a un mucopolisacarido de membrana presenta alta tendencia para adherirse a los cuerpos extraños ${ }^{10}$.

Cuando existe infección al igual que el resto de autores casi siempre retiramos la prótesis administramos antibióticos y esperamos 6 meses antes de valorar la recolocación. Mulcany retira 
la prótesis e irriga la zona con un protocolo de 7 soluciones antisépticas esperando también 6 meses antes de recolocar otra prótesis.

El grado de satisfacción general del paciente y de la pareja con el implante de la prótesis es alto y se sitúa en torno al $80 \%$ para el paciente y el $60 \%$ para la pareja guardando las causas de insatisfacción relación con falsas expectativas por parte del paciente o con complicaciones que dificultaron el manejo adecuado de la prótesis.

\section{CONCLUSIONES}

1. Las prótesis de pene continúan siendo un tratamiento eficaz para la disfunción eréctil en pacientes que no responden a tratamientos de primera o segunda línea.

2. Cuando aparecieron las nuevas terapéuticas orales hemos asistido a una disminución en el número de indicaciones disminuyendo el número de prótesis de causa vascular y aumentando las colocadas por cirugía previa.

3. La tasa de complicaciones es similar en nuestra serie al resto, siendo las más numerosas las derivadas de fallos mecánicos y las más graves la infección sobre todo en pacientes lesionados medulares y diabéticos

4. La tasa de satisfacción del paciente se sitúa en torno al 80\% estando el descontento sobre todo en relación con falsa expectativa por parte del paciente.

\section{REFERENCIAS}

1. Drogo K. Montague, Kenneth W. Angermeier. Las prótesis de pene en año 2000. AUA update series; 2:85-91.

2. Allona A, Sáenz de Tejada I. Erección, eyaculación y sus trastornos; 22:419.

3. Roselló Barbara M. Prótesis de pene. Indicaciones, clasificación, técnica, complicaciones y seguros. Arch Esp de Urol 1996;49(3):294-302.

4. Wilson SK, Cleves MA, Delk JR. Long-term followup of treatment for Peyronie`s disease: modeling the penis over an inflatable penile prosthesis. J Urol 2002;165:825-829.

5. Pereira Arias JG, Escobal Tamayo V, Maraña Fernández MT y cols. Implante de prótesis de pene en el tratamiento de la impotencia: nuestra experiencia. Arch Esp de Urol 1994;47(7):703-708.

6. Rodríguez Tolrá J, Franco Miranda E, Arbeláez Arango S y cols. Act Esp Urol 2002, abril:261-265.

7. John J. Mulcahy. Long-term experience with salvage of infected penile implants. J Urol 2000;163:481-482.

8. Villavicencio H, Parra L, Ruiz Castañe E. Tratamiento quirúrgico con prótesis de pene en 100 pacientes con disfunción eréctil. Act Esp Urol 1989 nov-dec;13(6):420424.

9. Chiang HS, Wu CC, Wen TC. 10 years of experience with penile prosthesis implantation in Taiwanese patients. $\mathrm{J}$ Urol 2000 feb;163(2):476-480.

10. Kimoto Y, Iwatsubo E. Penile prostheses for the management of the neuropatic bladder and sexual dysfunction in spinal cord injury patients: long-term follow up. Paraplegia 1994;32:336-339.

Dr. O. Rodríguez Faba

C/ Ámsterdam, 4 - 7ํㅗ

33011 Oviedo (Asturias)

(Trabajo recibido el 26 marzo de 2004) 